\title{
Performance Analysis of Iris Recognition System Using DWT, CT and HOG
}

\author{
Ajmi S J ${ }^{1}$, Prof. Arun P S ${ }^{2}$ \\ ${ }^{I}$ Dept. of Electronics and Communication Engineering Mar Baselios College of Engineering and Technology \\ Trivandrum, India \\ ${ }^{2}$ Dept. of Electronics and Communication Engineering Mar Baselios College of Engineering and Technology \\ Trivandrum, India
}

\begin{abstract}
Authentication and recognition can be achieved using biometric methods such as fingerprint, DNA, Iris etc. Human iris is the most reliable biometric because of its uniqueness, stability and noninvasive nature. The iris recognition system includes image acquisition, pre-processing (normalization and segmentation), feature extraction and recognition. For feature extraction, different methods considered are DWT, CT and HoG. $2 D D W T$, used to create a feature vector is decompose up to its $8^{\text {th }}$ level and the approximate coefficients are retained to represent the feature vector. Another transform technique namely contourlet transform (CT), which is a new $2 D$ extension of wavelet transform, employs the combination of laplacian pyramid and directional filter bank. The feature vector build using CT reflex the directional information of iris. This iris recognition also perform feature extraction using $H o G$. Whose feature vector is computed by dividing an iris image in to blocks and there after computing the gradient followed by histogram. The database used is CUHK and CASIA. These methods are evaluated based upon False Rejection Rate (FRR) and False Acceptance Rate (FAR).

Index Terms: Discrete Wavelet Transform (DWT), Contourlet Transform (CT), Histogram of Oriented Gradients (HoG), False Acceptance Rate (FAR), False Rejection Rate (FRR).
\end{abstract}

\section{Introduction}

Now a days biometric techniques for personal identification are more reliable as compared to traditional methods. The term "biometrics" is originated from the Greek words bio and metric, 'bio' means 'like' and 'metric' means 'to measure'. Most secure biometric personal identification methods based on physiological or behavioral characters are DNA, fingerprint, face, iris etc. They are mainly used in security and civilian applications. Iris based biometric identification technique is described here. Iris recognition is defined as the process of personal recognition by analyzing the iris pattern of that particular person. Iris recognition systems offer more accuracy than traditional personal identification methods like passwords, ID cards, ATM cards, these methods can be easily forgotten, lost or stolen. Iris is a part of eye, thin, circular in shape, present in between pupil and sclera. The eye color is specified by the color of iris. The size and diameter of the pupil is controlled by iris. Iris is unique to each person and to each eye. Over a life time the iris pattern is stable, so it provide noninvasiveness, high uniqueness, stability etc. Iris recognition is a complex process, it comprises of mainly four steps. Iris normalization, iris segmentation, feature extraction and recognition. The process of eliminating noise from iris image is iris normalization. Which is done by using different kinds of filters. Normalization is done at the first step because the presence of noise in the image leads to improper iris code. Iris segmentation is used to locate objects and boundaries in an image. Purpose of segmentation is to partition an image in to meaningful regions with respect to a particular application. Feature extraction is the key step in iris recognition system. Many methods are used in feature extraction are discrete wavelet transform, contourlet transform and histogram of oriented gradients. Wavelet transform splits the signal in to basis function sets, called wavelets. The single prototype, from which the wavelets are obtained is called mother wavelets. Wavelet analysis is used to represent functions or data. It is a mathematical technique used for nonstationary signal representation with high efficiency. Decomposition of wavelet is a multiresolution analysis, it decomposes images in to scaling functions and wavelet coefficients [1] [2] [3]. Contourlet transform (CT) is a new two dimensional non separable transform. CT is a new geometrical image transform, includes images of contours and textures. It is composed of Directional Filter Bank (DFB) and Laplacian Pyramid (LP). Laplacian Pyramid performs sub band decomposition of images and DFB analyzes the detail image [7]. Histogram of Oriented Gradient descriptors is based on local appearance and shape. HoG implementation can be achieved by dividing the image into cells and for each cell compiling a histogram of gradient directions [13] [14]. Calculate the feature coefficients from feature extraction and the feature vectors are formed by arranging the feature coefficients in vector form. Each feature vector is compared with the feature vector of image in the database. 


\section{Proposed System}

Iris recognition is defined as the process of personal recognition by analyzing the iris pattern of that particular person. Iris recognition systems offer more accuracy than traditional personal identification methods like passwords, ID cards, ATM cards, these methods can be easily forgotten, lost or stolen. Iris recognition is a complex process, it comprises of mainly four steps. Iris normalization, iris segmentation, feature extraction and recognition. General steps in iris recognition system are shown in Fig. 1.

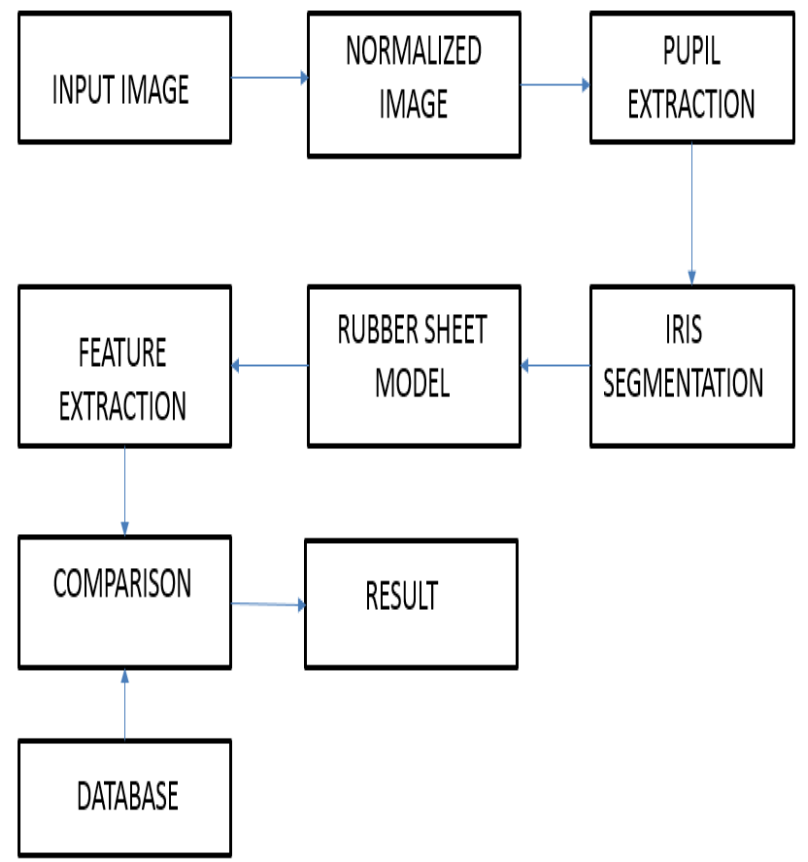

Fig. 1. Basic Block Diagram

First step is to capture the iris image, which is visible properly in the image. Pre-processing is applied on the input iris image for removing the noise.

\section{A. Iris Normalization}

Iris normalization is the process of removing noise from iris images by using different kinds of filters. Normalization is done at the first step because the presence of noise in the image leads to improper iris code. A Gaussian filter can be effectively used to remove such kind of noise from images. A Gaussian filter is considered to have the best trade of between noise removal and blurring.

\section{B. Pupil Extraction}

The darkest portion of eye is called pupil and it does not carry any information, so it can be removed. There are various steps involved in pupil extraction process. First one is image normalization, second step is grayscale to binary conversion, third step is edge detection. Canny edge detection is the best for iris application.

\section{Iris Segmentation}

Iris segmentation is used to locate objects and boundaries in an image. Purpose of segmentation is to partition an image in to meaningful regions with respect to a particular application.

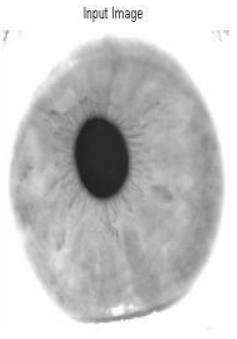

(a)

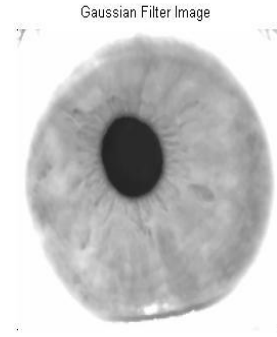

(b) 

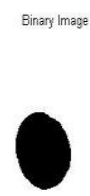

(c)
Edge Detected Image

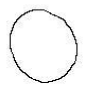

(d)

Fig. 2. (a) Input Image (b) Normalized image (c) Binary image (d) Edge Detected Image

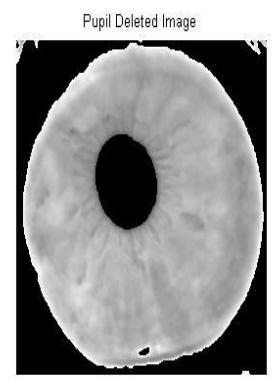

(a)

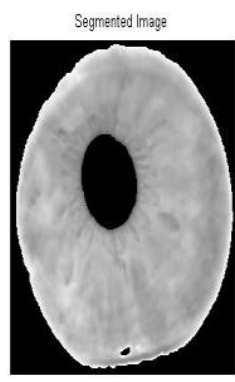

(b)

Fig. 3. (a) Pupil Deleted Image (b) Segmented Image

\section{Rubber Sheet Model}

Rubber sheet model is the rectangular format of iris. The steps involved are:

(i) Set the rotating arm at 0 degree position and select the direction of rotation.

(ii) Starting from 0 degree rotate the arm with suitable step size.

(iii) When a rotating arm is positioned at a particular angle from 0 degree, collect all the pixels along the rotating arm from R1 to R2 and arrange them in a column.

(iv) Move the rotating arm to next angular position, collect all the pixels along it from R1 to R2 and arrange them in the next column, in this way the desired portion of iris is scanned and rubber sheet is prepared [1].
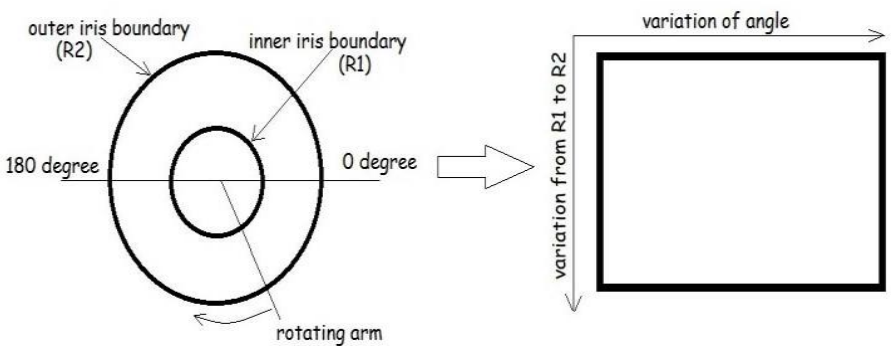

Fig. 4. Preparing a Rubber Sheet

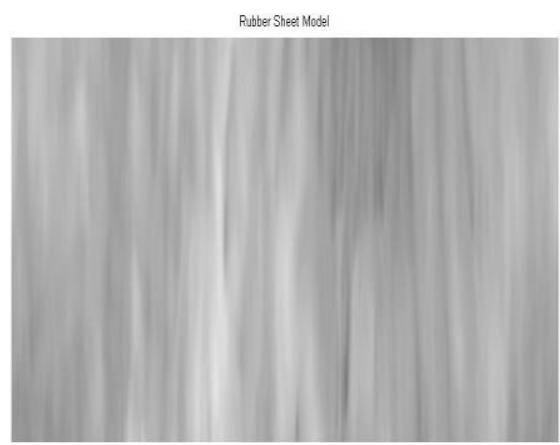

Fig. 5. Rubber Sheet Representation 


\section{E. Feature Extraction}

Feature extraction is the key step in iris recognition system. Many methods are used in feature extraction are discrete wavelet transform, contourlet transform.

\section{Discrete Wavelet Transform}

Wavelet transform splits the signal in to basis function sets, called wavelets. The single prototype, from which the wavelets are obtained is called mother wavelets. Wavelet analysis is used to represent functions or data. It is a mathematical technique used for nonstationary signal representation with high efficiency [2]. Decomposition of wavelet is a multiresolution analysis, it decomposes images in to scaling functions and wavelet coefficients. In DWT, signal energy is concentrates to specific wavelet coefficients. It converts images in to a series of wavelets. And it can be saved efficiently than pixel blocks. Representing signal in time scale is obtained by using digital filtering. The two dimensional transform decompose the original image into different channels, they are low-low, low-high, high-low, high-high. The decomposition can be applied to the low frequency channel (LL) to generate decomposition at the next level [3].

\section{Contourlet Transform}

The two dimensional extension of the wavelet transform is known as contourlet transform. It composed of directional filter bank and multiscale. The contourlet expansion contains basis images with different orientations in multiple scales and flexible aspect ratio. So it can effectively capture smooth contours. It is the combination of Laplacian Pyramid (LP) and Directional Filter Bank (DFB). Laplacian Pyramid performs sub band decomposition of images and DFB analyzes the detail image. DFB was designed to capture the high frequency directionality of the input image and it is poor on handling low frequency content, hence the DFB is combined with the LP, where low frequency of the input image is removed before applying DFB, where the whole spectrum is divided both angularly and radially and the number of directions is increased with frequency [7][17].

\section{F. Feature Code Generation and Comparison}

Rubber sheet output is divided into various blocks of sizes (8X8) and applying transforms on each block. Take average value of all the coefficients in each block for generating feature coefficients. All the feature coefficients arranged in vector form, are called as feature vector [1].

\section{Feature Vector Generation for DWT}

The rubber sheet output of iris image is divided into blocks and applying DWT on each block. Here DWT is applied up to $8^{\text {th }}$ decomposition level. Take only last approximate coefficient from each level and arrange them in vector form.

\section{Feature Vector Generation for CT}

Contourlet transform is applied on each block of rubber sheet output. CT is the combination of laplacian pyramid and directional filter bank. The filter bank outputs obtained are arranged in the form of vectors.

\section{Feature Extraction}

Another method of feature extraction is Histogram of Oriented Gradients (HoG). Which is directly applied on the grey scale iris image. Histogram of Oriented Gradient (HoG) are based on appearance. The feature vectors are used for window detection and computed appearance based features. Each window is divided in to $8 \times 8$ pixel cells and each group of cells of size $2 \times 2$ are integrated in to a block. These blocks are arranged in sliding manner so overlapping will occur. Each cells contain 9 bin HoG, concatenated vectors of cells are contains in each block. The HoG features are local geometrical changes and invariant to illumination, many recent studies have used them to detect iris. The descriptors of HoG has many advantages over other ones. Therefore HOG operates on all types of local cells. Histogram of oriented gradients are invariant to photometric and geometric transformations, except object orientation. This technique counts occurrences of orientation of gradients in all localized parts of interest and window detection. Algorithm: (1) Gradient computation (2) Orientation binning (3) Descriptor blocks (4) Block normalization [14].

The magnitude and phase equations are,

$$
\begin{aligned}
& M(x, y)=\operatorname{square} \operatorname{root}\left(f_{x}(x, y)^{2}+f_{y}(x, y)^{2}\right) \\
& \Theta(x, y)=\tan ^{-1}\left(f_{y}(x, y) / f_{x}(x, y)\right)
\end{aligned}
$$




\section{Result And Discussions}

To evaluate the performance of the proposed algorithm, we tested our algorithm on CUHK or CASIA database. Unlike fingerprints and face, there is no reasonably sized public domain iris database. On CUHK Iris Image Dataset from Hong Kong University that contains images from 36 persons with maximum 7 images from every one, we randomly choose 10 images and withdrew them from database in every test to have nonuniformly distributed data. The database used in the experimentation consists of 252 different iris images from CUHK iris image database. CUHK iris database is the only public domain database available. Size of each database iris image is $280 x$ 320. In this database there are 36 different subjects. Each subject has contributed to seven different images of same eye. Thus there are a total of 252 (36 x 7) images in the database. Among the seven images four are taken in first session and other three are taken in second session [8].

The time of computation for recognition process is tabulated in Table I. The time of computation is higher for DWT as compared to CT, and lesser for HoG as compared to the other two.

Table -Time Of Computation

\begin{tabular}{|l|l|}
\hline METHOD & TIME (Sec) \\
\hline DWT & 12.16 \\
\hline CT & 11.33 \\
\hline HOG & 8.33 \\
\hline
\end{tabular}

False acceptance rate is the measurement of how many imposter users are falsely accepted in to the system as genuine users. FAR is tabulated in Table II. FAR is lesser in HoG as compared to DWT and CT.

TABLE I. FAR

\begin{tabular}{|l|l|}
\hline METHOD & FAR $(\%)$ \\
\hline DWT & 0.78 \\
\hline CT & 0.78 \\
\hline HOG & 0 \\
\hline
\end{tabular}

False rejection rate is the measurement of how many genuine users are falsely rejected by the system as imposter. FRR is tabulated in Table III. FRR is lesser in HoG as compared to DWT and CT.

TABLE II. FRR

\begin{tabular}{|l|l|}
\hline METHOD & FRR $(\boldsymbol{\%})$ \\
\hline DWT & 1.1 \\
\hline CT & 0.78 \\
\hline HOG & 0.78 \\
\hline
\end{tabular}

Recognition rate is the measurement of how many users are correctly recognized from the total users. Recognition rate is tabulated in Table IV. Recognition rate is higher in HoG as compared to DWT and CT.

TABLE III. RECOGNITION RATE

\begin{tabular}{|l|l|}
\hline METHOD & Recognition Rate (\%) \\
\hline DWT & 98.02 \\
\hline CT & 98.4 \\
\hline HoG & 99.2 \\
\hline
\end{tabular}

\section{Conclusion}

In this thesis three different feature extraction methods for iris recognition were used such as DWT, CT and HoG. In DWT, Daubechies2 wavelet transform was approached with $8^{\text {th }}$ level decomposition for feature extraction and feature vector generation. By using this method an efficiency of $98.02 \%$ is achieved for 12.16 seconds. In CT, the combination of laplacian pyramid and directional filter bank, has an efficiency of $98.13 \%$ for 11.33 seconds which is better when compared with DWT. In HoG, $99.2 \%$ efficiency is obtained for 8.33 seconds. The results shows that the HoG has better recognition rate, lesser time of computation, less FAR, and less FRR as compared to the other two.

\section{References}

[1]. Ameya Deshpande, Sumitkumar Dubey, Hrushikesh Shaligram, Aditya Potnis and Satishkumar Chavan "Iris Recognition System using Block Based Approach with DWT and DCT," IEEE India Conference (INDICON), 2014.

[2]. D. Monro, S. Rakshit, and D. Zhang, "DCT-based iris recognition," IEEE Trans. Pattern Anal. Mach. Intell., vol. 29, no. 4, pp. 586$595,2007$. 
[3]. Daugman J., "New methods in iris recognition”, IEEE Trans. Syst., Man, Cybern. Part B: Cybern., vol. 37, no. 5, pp. 1167-1175, 2007.

[4]. LiYu, David Zhang and Kuanquan Wang, “The Relative Distance Of Key Point Based Iris Recognition,” Pattern Recognit., vol. 40, pp.423-430, 2007.

[5]. J. Daugman, "How iris recognition works," IEEE Trans. Circuits and Systems for Video Technology, Vol.14, No.1, pp. 21-30, 2004.

[6]. Amol D. Rahulkar and Raghunath S. Holambe "Rotation Invariant Iris Texture Verification," AKGEC Journal of Technology, Vol.1, pp. 1-6, 2010.

[7]. Amir Azizi and Hamid Reza Pourreza "Efficient IRIS Recognition Through Improvement of Feature Extraction and subset Selection", (IJCSIS) International Journal of Computer Science and Information Security, Vol. 2, No.1, June 2009.

[8]. CASIA, "Chinese Academy of Sciences - Institute of Automation". Database of 756 Grayscale Eye Images. http://www.sinobiometrics.com Versions 1.0, 2003.

[9]. A.K. Jain, R.M. Bolle, and S. Pankanti, Eds. "Biometrics: Personal Identification in a networked Society", Norwell, MA: Kluwer, 1999.

[10]. J. Daugman, "Biometric personal identification system based on iris analysis," 1994, US patent no. 5291560.

[11]. Panchamkumar Shukla, "Complex wavelet transform and their applications," Signal Processing Division, Department of Electronic and Electrical Engineering, University of Strathclyde, Glasgow, Chapter no.2, pp. 16-14, 33-36 and 83-85, 2003.

[12]. L.V. Birgale and M. Kokare, "Iris Recognition Using Discrete Wavelet Transform", IEEE Proceedings of the International Conference on Digital Image Processing (ICDIP'09), 2009.

[13]. H. Wang and S. F. Chang, "A Highly Efficient System for Automatic Face Region detection in MPEG video", IEEE Transactions on Circuits and Systems for Video Technology, Vol. 7, No. 4, pp.615-628, 1997.

[14]. W. T. Freeman and M. Roth, "Orientation histograms for hand gesture recognition", Intl. Workshop on Automatic Faceand GestureRecognition, IEEE Computer Society, Zurich, Switzerland, pages 296.301, June 1995.

[15]. K. D. Baker and G. D. Sullivan, "Multiple bandpass filters in image processing," IEEE, vol. 127, pp. 173 -184, 1980.

[16]. Peter J. Burt and Edward H. Adelson, "The Laplacian Pyramid as a Compact Image Code", IEEE Transactions on Communications, Vol. Com-31, No. 4, April 1983.

[17]. Yue Lu and Minh N. Do, "Multidimensional Directional Filter Banks and Surfacelets", IEEE Transactions on Image Processing. 